\title{
Miranda
}

Revue pluridisciplinaire du monde anglophone /

Multidisciplinary peer-reviewed journal on the English-

speaking world

$8 \mid 2013$

In Umbra Voluptatis : Shades, Shadows, and their

Felicities / Film Adaptations, New Interactions

\section{Ghost World(s) - couleur, espace, verbe et esthétique}

\section{Nicolas Labarre}

\section{(2) OpenEdition}

Journals

Édition électronique

URL : http://journals.openedition.org/miranda/3363

DOI : 10.4000/miranda.3363

ISSN : 2108-6559

\section{Éditeur}

Université Toulouse - Jean Jaurès

Référence électronique

Nicolas Labarre, «Ghost World(s) - couleur, espace, verbe et esthétique », Miranda [En ligne], 8| 2013, mis en ligne le 28 juin 2013, consulté le 16 février 2021. URL : http://journals.openedition.org/miranda/ 3363 ; DOI : https://doi.org/10.4000/miranda.3363

Ce document a été généré automatiquement le 16 février 2021.

\section{(c) $)(9)$}

Miranda is licensed under a Creative Commons Attribution-NonCommercial-NoDerivatives 4.0 International License. 


\title{
Ghost World(s) - couleur, espace, verbe et esthétique
}

\author{
Nicolas Labarre
}

\section{Introduction}

1 Film de Terry Zwigoff sorti en 2001, Ghost World est d'abord un roman graphique du scénariste et dessinateur Dan Clowes publié en 1998, centré sur le mal-être et la quête d'identité d'Enid Coleslaw et de son amie, Rebecca Doppelmeyer. Les deux jeunes filles viennent de terminer leurs études au lycée, commentent avec acidité le monde qui les entoure et vont peu à peu s'éloigner l'une de l'autre. Rebecca choisit de s'intégrer, avec un travail, un compagnon, tandis qu'Enid se résout à fuir, prenant le bus pour une destination inconnue dans la dernière case du récit. En accord et en collaboration avec Clowes, Zwigoff a fait de ce récit un film qui ne se contente pas de reprendre les éléments narratifs de l'original - personnages, situations et dialogues - dont la transposition est relativement non-problématique (Hutcheon 11-14). Il n'a pas non plus choisi de pousser la logique de l'adaptation jusqu'à une recréation de l'esthétique de la bande dessinée d'origine, comme ont pu le faire des films aussi différents par ailleurs que Dick Tracy (Beatty 1990), Hulk (Lee 2003) ou Sin City (Rodriguez et Miller 2005). Plus que la fidélité à un original, le film vise à mettre en œuvre une série de variations sur les bases mises en place par Clowes. Ainsi, sans renoncer à la construction d'une esthétique propre, le long-métrage intègre dans sa structure et ses choix stylistiques certaines des options formelles du roman graphique. Ces mécanismes, qui ont trait en particulier à la représentation de l'espace, sont intégrés dans une esthétique et des dispositifs cinématographiques par ailleurs très classiques, mais aussi relayés et complétés par l'investissement de champs stylistiques absents de la bande dessinée d'origine.

2 Cet essai s'intéresse à cette transposition partielle de ces modes de représentation d'un médium à l'autre, en tentant de rendre compte des effets de ce déplacement au sein d'un ensemble d'outils et de conventions différents. Il s'agira donc d'expliquer en quoi 
le film constitue une expérience de consommation culturelle fort éloignée du roman graphique d'origine, même en tenant compte de ce que Pascal Lefèvre a appelé les « ontologies incompatibles » des images des deux médias (Lefèvre 2007).

\section{Adaptation et renforcement narratif}

3 Ghost World ${ }^{1}$ est bien un roman graphique, dans la mesure où le terme renvoie à des récits ambitieux, perçus comme littéraires, et ne relevant pas seulement du divertissement populaire (Gabilliet). Ainsi, il ne s'agit pas d'une bande dessinée de genre, elle est centrée sur le développement de personnages psychologiquement complexes, elle est l'œuvre d'un auteur singulier, et elle n'est pas non plus entièrement assujettie au règne de la narrativité. Seules les traces de sa publication sérielle rapprochent GWGN des formes de culture de masse. Le récit est en effet à l'origine une suite de huit épisodes, publiés par Fantagraphics dans le comic book de Dan Clowes Eightball, de juin 1993 ( $\left.n^{\circ} 11\right)$ à mars 1997 ( $\left.n^{\circ} 18\right)$. Si ces épisodes sont clos sur euxmêmes, relativement autonomes, ils dessinent dans leur totalité un arc narratif identifiable, décrivant la dissolution de la relation d'amitié liant Rebecca et Enid. GWGN, comme les autres œuvres de Clowes jusqu'à Wilson (2010), porte donc la marque d'une fragmentation, d'une structure épisodique, parcourue de discontinuités.

GWGN pose donc immédiatement un problème en termes d'adaptation pour le cinéma narratif classique. Le premier travail d'unification est mené par Clowes au moment de rassembler ses épisodes sous forme de livre, puisqu'il uniformise les couleurs des différents chapitres, reprend certains dessins de visages, renomme les épisodes «chapitres» et introduit un péritexte complétant en quelques images la vie des personnages. Interrogé à la sortie du film, Zwigoff déclarait pourtant qu'à sa première lecture du roman graphique, il n'y avait pas vu d'intrigue, seulement des dialogues pertinents («Subtlety Gap Can Be Hard to Fill »). L'adaptation est donc d'abord liée à une consolidation du récit, ce qui amène par exemple un élément du péritexte de la version collectée, la cérémonie des diplômes, à être intégré au scénario du film. Comme une confirmation de ce processus de consolidation et d'étoffement, de nombreuses scènes ne proviennent pas de Ghost World proprement dit, mais de courts récits de Clowes publiés également dans Eightball (dont «Art school confidential» pour le cours d'arts plastiques, «Gynecology » pour l'épisode du poster raciste, «Feldman » pour la scène du quizz dans le café). ${ }^{2}$ Surtout, le film ajoute à la structure de GWGN un personnage clé, Seymour, un adulte collectionneur de vieux disques de jazz qui altère sensiblement la dynamique des rapports amicaux et amoureux de l'original. GWF pratique donc simultanément une élision et un étoffement de la trame par le recours à des éléments exogènes au récit. Si certaines scènes du film tiennent encore de l'épisode isolé, celui-ci propose néanmoins une progression dramatique classique, avec un début, une fin et trois actes identifiables, dont la durée même est conventionnelle. L'amitié des deux jeunes filles face à un monde fantôme est mise en danger par la rencontre avec Seymour, après trente minutes. L'amitié ambiguë d'Enid avec celui-ci débouche sur une consommation, après une heure vingt-cinq de film. Enfin, le troisième acte met en scène en vingt-cinq minutes la rupture, la jalousie de Seymour et le départ d'Enid après une esquisse de réconciliation.

Ces contraintes narratives ne sont pour autant pas le seul facteur différenciant les deux versions de Ghost World. Une adaptation littérale, en admettant qu'elle soit possible, 
aurait dans toutes les hypothèses débouché sur un objet qui, par son contexte de production et de réception, aurait été vu et perçu de façon nécessairement différente $\mathrm{du}$ roman graphique. Le rapport au genre induit ainsi une distance entre les deux versions, du point de vue des attentes et des références convoquées. Le genre du teen movie est consacré et traite fréquemment du sentiment d'aliénation de ses protagonistes, avec plus ou moins de réalisme, de Rebel Without a Cause (Ray 1955) à Saturday Night Fever (Badham 1977) ou, plus près de nous, The Rules of Attraction (Avary 2002). Dans la bande dessinée nord-américaine contemporaine, le genre est moins fréquenté. Les histoires adolescentes y ont longtemps été racontées sous l'angle de l'autobiographie, chez Chester Brown ou Joe Matt par exemple, ou par un recours au fantastique comme métaphore, de Spider-Man au Black Hole de Charles Burns; les romance comics, genre central de la fin des années quarante, ont quant à eux totalement disparu. Dans le champ de la bande dessinée indépendante, ${ }^{3}$ GWGN apparaît donc comme un objet singulier, indépendant du réseau d'attentes et de promesses, stylistiques ou narratives qui s'attache au genre cinématographique. Le niveau d'attente supplémentaire qu'est le genre (supplémentaire, car la forme du medium et le corpus de l'auteur entre autres orientent nécessairement la lecture) affaiblit ou renforce certains effets lors du passage de la page à l'écran (Lefèvre 2011). Le badinage sexuel entre Josh et Rebecca, par exemple (à la page 56 et à 27 minutes) souffre de la présence intertextuelle des sex-comedies à la American Pie, sous-genre des teen movies populaire mais déconsidéré. Les affiches qui vantent une "comédie", soulignent d'ailleurs cette emprise du genre, et en creux son absence dans la bande dessinée de Clowes. A cette question du genre s'ajoutent les paramètres intertextuels liés aux acteurs. Thora Birch venait juste de jouer une adolescente aliénée dans American Beauty (Mendes 1999), succès critique et populaire, et les contrastes entre ce personnage et celui qu'elle joue dans GWF prennent de ce fait un relief particulier. Plus encore que la question du genre, cependant, ces paramètres intertextuels sont à historiciser. Le sens de la présence de Scarlett Johansson, à la lumière rétrospective de Lost in Translation (Coppola 2003), est très différent de ce qu'il pouvait être à la sortie du film, époque à laquelle elle était principalement connue pour son personnage juvénile dans The Horse Whisperer (Redford 1998).

6 Pour autant, certains indices attestent de la ressemblance entre les deux versions dans leurs usages et leur réception, ressemblance qui ne se laisse pas résumer par la trompeuse équivalence entre cinéma indépendant et bande dessinée indépendante. ${ }^{4}$ Confirmant le jugement de Linda Hutcheon, pour qui le plaisir de l'adaptation est celui d'une " répétition sans duplication » (173), le succès du film continue à nourrir celui de la bande dessinée (Clowes 7-8). La dernière édition en date de GWGN aux Etats-Unis ajoute d'ailleurs le script et des photos de production au roman graphique, en un objet cartonné et coûteux, qui suppose que le public de l'un est aussi le public de l'autre. L'attrait de ce rapprochement tient moins à la découverte d'un « original » que d'une exploration de variations sur un thème..$^{5}$

7 Les contraintes narratives, le rôle du genre et de l'intertextualité sont autant d'éléments qui rendent impossible une transposition littérale du roman graphique de Clowes. Cependant, à l'intérieur de ces contraintes, celui-ci offre un terrain apparemment propice à une adaptation. La simplicité de ses dispositifs visuels et structurels, ainsi que sa relative "transparence », suggèrent une compatibilité avec les modes de représentations cinématographiques. 


\section{Compatibilité des modes de représentation}

8 S'il a souvent été souligné que la bande dessinée partage de nombreux codes et procédés avec le cinéma en termes de représentation et de découpage, ce constat est surtout vrai, dans le domaine nord-américain, pour les comic strips à vocation réaliste et leurs descendants stylistiques. Dans ce cas, comme dans la tradition hollywoodienne décrite par Bordwell et Thompson, un objectif commun de "transparence » rapproche les deux systèmes de représentation. Les similitudes entre les deux médias sont bien plus rares pour de nombreux comic strips se déroulant dans un espace conventionnel et ne prétendant pas au réalisme, à l'image de Krazy Kat de Herriman ou Peanuts de Schulz. Elles le sont également pour une bonne part de comics parmi ceux que le critique Charles Hatfield qualifie collectivement d'« alternatifs » (Hatfield 3-31), dans lesquels il inclut Ghost World (155). Ces comics, qui correspondent assez étroitement au type de récits auxquels renvoie la notion de graphic novel, tendent à proposer une représentation de l'espace et une mise en page ne visant pas la mimesis, mais l'expressivité ; les travaux de Chris Ware, comme Jimmy Corrigan, en sont l'exemple le plus notable. Ceux-ci jouent fréquemment de la page de bande dessinée comme surface tabulaire et pas seulement comme le site d'une lecture linéaire, en offrant plusieurs parcours de lecture, en soulignant la coprésence des cases plutôt que leur séquence (Hatfield 48, Fresnault-Deruelle 16-23). Si cette approche tabulaire est présente dans d'autres œuvres de Clowes (Schneider), elle n'est que peu mobilisée dans GWGN, et lorsqu'elle affleure, c'est comme supplément possible mais non nécessaire d'une lecture linéaire (page 19, par exemple). Cette linéarité est un élément probant de compatibilité avec la forme cinématographique.

9 La grille qui structure les planches de GWGN est des plus classiques, avec des pages comprenant trois strips, et entre une et trois vignettes par strip. Là se constitue un espace narratif au sein duquel de nombreux gages de réalisme sont donnés, puisque cet espace est cohérent, stable, et donne l'illusion de profondeur dans la surface plane de la page. Les variations sur cet ordonnancement existent, mais restent contenues, avec des déviations mineures dans moins d'un quart des planches. Ainsi, le chapitre quatre oppose un récit au présent, utilisant quatre strips par page et un récit rapporté, qui utilise le format des trois strips par page. Encore ce récit est-il surcodé par l'absence de filet de contour autour des cases, ainsi que par la présence d'une narration au dessus des illustrations. Une scène de phantasme du chapitre 3 (32), quant à elle, supprime le filet, bénéficie d'une typographie différente (là encore, surcodage d'un événement très lisible), mais respecte la grille. Les premières pages de chapitre sont également l'occasion de variations mineures sur cette grille, qui permettent l'inclusion d'un titre (bandeau latéral, case unique dans un strip), sans pour autant remettre en cause le système dans son ensemble. Ce classicisme est en lui-même signifiant, puisqu'il se présente comme un choix et non plus comme une convention ou une directive éditoriale, comme ce pouvait être le cas dans la bande dessinée classique (Groensteen 112-113). Cette grille vient ici renforcer les grands thèmes du récit, en particulier le poids du passé, le délabrement mais la persistance des codes et conventions, ou encore la monotonie de l'existence et le déterminisme. Cette régularité choisie produit des cases et des séquences au cadre rectangulaire, à la mise en page signifiante mais 
discrète. Le code est relativement transparent et ses effets de sens sont réservés au lecteur percevant le caractère délibéré de la régularité affichée.

La même observation pourrait s'appliquer aux textes, puisqu'en dehors des titres et des éléments de décor, le texte dans Ghost World se limite à des paroles dans des bulles : le récit ne comporte ni récitatif, ni bulles "de pensées", ni même d'onomatopées inscrites dans l'image. S'il n'y a pas homologie entre la bulle et le dialogue de cinéma (le caractère conventionnel de la bulle est plus évident, sa trace graphique masque en partie le décor), tout du moins $\mathrm{y}$-a-t-il une transposabilité relativement non problématique de ces dialogues. En d'autres termes, si Ghost World n'est en rien un storyboard, il présente au niveau de la planche, du récit et de l'interaction texte-image, un ensemble de caractéristiques qui le rendent adaptable sans avoir à réinventer le récit, en cherchant seulement des transpositions parmi un ensemble de procédés éprouvés, connus au moins depuis l'époque des serials (Labarre).

\section{L'espace dans le roman graphique}

11 C'est donc dans le contenu des cases plus que dans leur organisation sur la page que se situe la spécificité de GWGN, et en particulier dans l'isolement des personnages au sein d'un espace par trop géométrique. La grille régulière de GWGN, évoquant la carte d'une ville américaine au cordeau (Venezia), est à l'image de l'espace contenu dans les cases. La géométrie s'impose dans des espaces parfaitement construits, à l'implacable régularité. L'étroitesse des cases, et leur format allongé mènent fréquemment des perspectives extrêmement fuyantes, alternant avec des compositions dans lesquelles les objets s'alignent parfaitement avec les bords du cadre. La géométrie n'est alors pas seulement un mode de représentation, elle délimite un espace ostensiblement mathématique et artificiel. Le premier chapitre propose ainsi une exploration sur six pages de la chambre de Rebecca, multipliant les angles de vue pour reconstruire en totalité un espace cohérent. Le trait de l'auteur est rigide, imitant le rendu et la finesse des stylos calibrés de type Rapidograph, et seule une inspection détaillée révèle l'usage du pinceau, littéralement neutralisé. La mise en place de surfaces noires et blanches ne relevant pas d'un jeu de lumière naturaliste vient encore renforcer ce caractère abstrait d'un monde aux lignes acérées. Ces ombres noires sont absentes du dessin des personnages, qui gagnent quant à eux du volume par un travail de feathering, des hachures adoucissant bords et arêtes. Le choix d'utiliser des traits de pinceau souples pour les contours de ces personnages, qui crée une opposition graphique à l'intérieur de la case, souligne la fonction rhétorique de la représentation géométrique et austère des lieux. Précisons qu'il s'agit bien là d'un choix, puisque dans ses travaux précédents (Lloyd Llewellyn, surtout), Clowes faisait usage d'un trait bien plus anguleux pour les personnages.

12 La géométrie des décors fait donc écho à la grille régulière qui les contient, et s'oppose au graphisme plus souple des personnages. Cet usage conjoint de la grille et de la géométrie n'est pas une invention de Clowes. Dans les fascicules de la firme E.C. des années 1950, que l'auteur de Ghost World cite comme une influence importante (Hignite 168), Harvey Kurtzman avait déjà mis en lumière ces potentialités de renforcement de la structure de la page et de la composition des cases. Il s'agissait de confronter l'humain à un environnement rendu anonyme et inhospitalier par la commercialisation, en enfermant par exemple un consommateur dans une grille 
reproduisant la structure des rayons alignés (« Supermarkets », dans Mad n 19, janvier 1955). Ce n'est donc pas ce codage qui est singulier dans GWGN, puisqu'on en trouverait trace dans une œuvre aussi différente du récit de Clowes que le Brüsel, de Schuiten et Peeters, mais son usage répété, systématisé au cours du récit. Une brève échappée vers la mer y prend alors un caractère de rupture graphique majeure, quand s'imposent des formes douces et irrégulières dans le décor, tracées d'un trait de pinceau identifiable comme tel (77-78).

13 Ce monde fantomatique n'est pas seulement grillagé, il est également placé sous le règne de la transparence. Clowes multiplie les représentations de ses personnages à travers des fenêtres, dans des chambres, des diners ou des voitures. Ces vitres ne se laissent pas voir comme des surfaces éventuellement réfléchissantes, comme des miroirs possibles permettant le retour sur soi, mais comme des ouvertures béantes à la transparence parfaite. Clowes n'utilise en effet que très rarement les multiples conventions de représentation (aplat de couleur, traits de pinceau, reflets esquissés) permettant de distinguer en bande dessinée une fenêtre ouverte d'une fenêtre fermée. Tony Venezia a suggéré que l'espace dans Ghost World était composé de "non-lieux ", "non places", c'est-à-dire des endroits qui ne sont connectés ni à l'histoire, ni à des questions identitaires, ni aux relations interpersonnelles (Venezia). De fait, ces « nonlieux » sont fort peu peuplés : les perspectives ouvertes le sont sur des espaces vides. A contrario, l'opacité et le désordre prennent une valeur positive à plusieurs reprises dans le récit, qu'il s'agisse de la chambre d'Enid ou du pourboire conséquent qu'elle abandonne sur une table de diner après un épisode peu reluisant. Les graffitis qui viennent perturber cet espace trop ordonné pour y inscrire une expression énigmatique ("Ghost world ») relèvent de la même tactique de résistance.

La principale source d'opacité dans le récit provient des bulles. Arrondies, souples, lettrées manuellement et parcourues d'irrégularités (en gras), elles masquent en partie les perspectives trop parfaites. Surfaces opaques, dont la platitude s'oppose violemment à la tridimensionnalité suggérée de l'espace diégétique, elles sont, selon l'expression de Pierre Fresnault-Deruelle «cette présence blanche qui neutralise le décor» (Groensteen 82). Cette opacité dissimule la laideur du monde fantôme, et les dialogues que contiennent ces bulles fonctionnent à l'unisson. C'est par les mots qu'Enid notamment s'évertue à redonner une signification à des lieux-simulacres (l'Amérique de Ghost World est aussi celle de Baudrillard) comme une fausse cafeteria années 1950 (39) ou un parc de copies médiocres des Flintstones (73).

A en croire Terry Zwigoff, ce sont ces dialogues qui l'ont poussé à adapter Ghost World. Il se montre de fait particulièrement peu disert lors d'entretiens sur la dimension graphique de l'ouvrage et sur son éventuelle transposition, alors que par son titre même, la bande dessinée met en exergue la question de la représentation de l'espace. La photographie du film, confiée à Affonso Beato, renommé pour ses collaborations avec Almodovar, n'est pas du ressort de Clowes, qui devient seulement un artiste du mot en coécrivant le scénario avec Zwigoff. Pourtant, le traitement de l'espace est si prégnant dans GWGN que la version filmée porte les traces de certains des choix effectués pour la bande dessinée. 


\section{L'espace dans le film}

16 Le monde mathématique et géométrique mis en place par Clowes n'est ainsi pas absent du film. Si le cinéma hollywoodien classique rechigne à afficher la géométrie de ses décors (Bordwell, Staiger et Thompson 53), Zwigoff souligne a contrario volontiers le rectangle du cadre, en y inscrivant des lignes qui lui sont parallèles. Les plans de situation (establishing shot), en particulier, alternent entre des cadrages frontaux (7'35, $\left.13^{\prime}\right)$ et des perspectives délibérément fuyantes (1'12), et sont pour certains répétés à l'identique à plusieurs reprises dans le cours du film. A l'intérieur même des séquences, GWF utilise les possibilités graphiques des enfilades de sièges, multiplie les cadrages frontaux ou les discussions entre personnages installés de façon parfaitement orthogonale à l'angle de prise de vue. A cela s'ajoute d'autres dispositifs, comme l'usage répété d'un motif géométrique (carreaux, bouteilles) bloquant le fond du plan, tandis qu'une grande ouverture focale neutralise la perspective. Si certaines scènes sont quasiment identiques dans le film et dans la bande dessinée (la visite à l'appartement de Josh à la page 56 et à 27 minutes), on peut parler dans l'ensemble de renversement du dispositif, pour obtenir un effet similaire d'un medium à l'autre. En effet, GWGN se distingue surtout par sa façon d'imposer une tridimensionnalité soulignée à l'espace plan de la page. Le film, quant à lui, malgré les quelques exemples de perspectives mentionnées précédemment, se singularise par la multiplication des espaces apparemment plans. Dans les deux cas, l'espace apparaît comme construit, artificiel, distinct des personnages qui l'occupent.

17 La thématique de la transparence, en revanche, ne joue pas le même rôle dans le film que dans le roman graphique. Si le film utilise bien quelques plans à travers une vitre, il ne systématise pas le procédé. Plusieurs explications peuvent être suggérées. L'une tient à la disparition de la possibilité de jouer du contraste entre opacité du verbe (la bulle) et transparence du monde. La matérialité des décors rend également difficile la reproduction du dessin de Clowes, dont les bâtiments sont tout en surface et sans épaisseur. Enfin, cette esthétique de la transparence prend indéniablement un sens différent dans le paradigme cinématographique, puisque son utilisation abondante renvoie à un intertexte qui est plus celui du voyeurisme que celui de la révélation, que l'on pense à Rear Window (Hitchcok 1954) ou aux slasher movies sur le modèle de Halloween (Carpenter 1978).

Cependant, l'écart dans la représentation de l'espace entre les deux versions tient avant tout au fait que, dans le film, la géométrie dans le plan n'est pas relayée par le système architectural de la grille. Celle-ci relève, nous l'avons dit, d'une variation sur un système canonique, la grille semi-régulière: elle est productrice de sens sans être ostentatoire. Or, le film ne peut rendre son cadre signifiant, à l'intérieur d'un système d'options transparentes dans un cinéma grand public. S'il existe des procédés permettant de modifier la forme du cadre, comme les caches, le split screen, ou le masque partiel par un premier plan abstrait, ceux-ci s'imposent comme des déviations par rapport à la norme, des procédés susceptibles de détourner l'attention sur eux plutôt que sur leurs effets (que l'on pense à Timecode de Mike Figgis, 2000, par exemple). Contrairement à Hulk (Lee 2003) ou au récent Scott Pilgrim vs. the World (Wright 2010), GWF ne cherche pas à représenter la bande dessinée, mais à en donner une lecture cinématographique qui ne soit pas ostensiblement dépendante d'un «original». Le réalisateur se tient à l'écart des procédés renvoyant à la forme de la source, révélant le 
caractère transmédiatique du récit : la relative transparence du medium qui caractérise le roman graphique est un trait que partage le film. Le travail formel se fait donc dans le cadre et dans le montage plus que sur le cadre.

La différence de format qu'induit le ratio 1,85:1 par rapport aux cases souvent carrées ou verticales de GWGN conduit à une représentation de l'espace fort différente. Les cases étroites de Clowes, largement occupées par le corps de ses principaux protagonistes, lui permettent de présenter un monde dépeuplé, mais sans ostentation. Si les bars et diners semblent quasiment déserts dans la bande dessinée, la verticalité permet plusieurs lectures possibles: le cadre comme reflet de la relation exclusive entre Enid et Rebecca, comme mode d'exclusion de personnages secondaires nonsignifiants, ou comme modèle réduit de la vacuité de l'espace diégétique tout entier. Il n'y a pas de hors-cadre en bande dessinée (Groensteen 50), et le dispositif véhicule une ambiguïté sur l'espace représenté, puisque celui-ci ne nous est présenté que sous forme fragmentaire. Dans le cours de la narration, le lecteur a rarement à trancher quant à la présence ou non d'autres occupants en ces lieux, sauf dans le cas du faux-diner années 1950, présenté comme désertique. Même dans une rare scène de "foule », l'exiguïté du cadre suggère un espace occupé par quatre ou cinq personnes seulement en dehors des principaux protagonistes (64). Dans son roman graphique précédent, le très lynchien Like a Velvet Glove Cast in Iron, les cadrages plus lointains permettaient à Clowes de présenter un monde objectivement désertique. Dans GWGN, ce désert devient une possibilité de lecture parmi d'autres. Le film, qui construit ses scènes en alternant des plans généraux et des cadrages plus rapprochés, dans un cadre toujours horizontal, n'offre pas cette ambiguïté, et les lieux sont peuplés de façon prévisible. Zwigoff attire d'ailleurs parfois l'attention sur les figurants, comme contrepoint du premier plan (à 47 minutes 52, par exemple). On peut donc parler de repeuplement du monde fantôme, d'autant que la narration inclut également plusieurs lieux très peuplés, en particulier une scène dans un bar à concerts, sans équivalent dans le roman graphique.

\section{De l'espace au récit}

20 Ce changement dans la représentation de l'espace est lié à une modification de la dynamique de la narration, mais aussi de la caractérisation des personnages : le récit, au sens étroit du terme, est dépendant du système de représentation qu'il emprunte (Hutcheon 9-15). L'Enid de Clowes se désolait de voir son endroit préféré envahi par les touristes (64), celle de Zwigoff est bien moins hostile à la foule et aux lieux publics. Elle entraîne Seymour, le solitaire, dans un bar bondé, où il finit bousculé, maudissant l'ignorance d'amateurs de ce qu'il faut bien appeler une culture de masse. A l'approche binaire (Enid/Rebecca) du roman graphique, où un triangle amoureux avec Josh - un jeune homme de leur âge - n'est qu'esquissé, le film substitue une approche ternaire (Rebecca/Enid/Seymour).

21 Si le roman graphique offre une vision unique de l'espace grillagé du monde fantôme, commune aux deux jeunes filles, le film oppose deux types d'espace, celui de Seymour et celui de Rebecca, en plaçant le personnage d'Enid à leur charnière. Seymour est associé à l'enfermement, à la solitude, à l'individualité forcenée, tandis que Rebecca se voit rejetée du côté d'une certaine conventionalité, du côté des lieux de passage (le travail, le bar) et de l'extérieur. Ce système d'oppositions est aussi lié à l'abandon du thème de la transparence systématisée, ce qui permet d'opposer l'appartement fermé 
et mal éclairé de Seymour au bow-window donnant sur la rue de celui choisi par Rebecca. Enid apparaît à plusieurs reprises comme une médiatrice déçue entre ces espaces incompatibles: elle emmène ainsi sans succès Rebecca chez Seymour et réciproquement, son expédition au bar avec ce dernier est un fiasco.

Là où la bande dessinée propose des lieux déconnectés les uns des autres, mais présentant une forte cohérence interne, le film met en place un espace moins systématiquement exploré, mais dans lequel il est possible de circuler. Au cours d'une longue scène au cours de laquelle Enid guide Seymour en ville, la cohérence de l'espace urbain apparait clairement : le sex-shop est derrière le bar, celui-ci fait face à l'épicerie de Josh, et tous ces lieux sont proches de l'arrêt de bus désaffecté où se conclut le film (de 40 minutes 30 à 42 minutes 30 environ). Ce parcours à pied s'oppose de façon révélatrice à une problématique récurrente de GWGN, la nécessité d'obtenir un véhicule, un chauffeur ou d'apprendre à conduire pour se rendre d'un lieu à un autre (43, 66 et 68). Les lieux individuels de la version filmée sont également moins importants, moins chargés d'affect, ils sont aussi parcourus de façon moins exhaustive (la géographie de l'appartement de Seymour reste mystérieuse) : leur multiplication les rend moins significatifs, moins ambigus, et leur rôle dans le récit tient essentiellement aux contrastes ou aux échos existant entre eux et les personnages principaux. L'unification du récit, une intrigue unique plutôt que huit chapitres semi-autonomes et des récits auxiliaires, liés à des lieux distincts, s'accompagne donc d'une unification ou plutôt d'une mise en continuité de l'espace. Une esthétique du fragment, qui souligne que le monde fantôme est exclu de l'histoire, est remplacée par une esthétique de la circulation et de la tension, qui en altère le sens en introduisant la notion de choix, d'alternative.

Cet espace reste donc malgré tout signifiant. Après avoir balancé longtemps entre les valeurs de Seymour (intérieur, solitaire, vieux) et celles de Rebecca (extérieur/ ouverture, public, moderne), dans un jeu d'adhésion, de refus et finalement de fuite, Enid opte pour une synthèse personnelle. L'échappatoire qu'elle se choisit emprunte à la valeur de solitude de Seymour, mais fait partie d'un système de circulation public, un bus, dont l'ouverture et la transparence même renvoient à ce qu'incarne Rebecca dans le film. Cette valeur de synthèse en même temps que de renoncement est absente de la bande dessinée, notamment parce que le jeu d'opposition s'y construit directement entre Enid et Rebecca, sans réel contrepoint.

\section{La couleur dans le film Ghost World}

24 L'écart maximal entre les deux versions de Ghost World ne se situe cependant pas dans la gestion de la géométrie de l'espace diégétique (et ses conséquences narratives), mais dans le travail sur la couleur. Cet écart est flagrant dès l'affiche du film, qui présente les deux héroïnes sur fond blanc, vêtues de vêtements aux couleurs saturées. Les images qui accompagnent le générique d'ouverture confirment cette impression, avec une palette de couleurs vives aux contrastes violents, encore renforcée par l'emprunt à une comédie musicale bollywoodienne, tout en rouge et doré.

GWGN se présente en noir et blanc, seulement rehaussé d'une couleur unique. Lors de la publication sérielle, cette couleur unique variait de numéro en numéro, mais dans la version collectée, il s'agit d'un bleu-vert pour la totalité des chapitres. La fonction de cette couleur est incertaine, comme le souligne la comparaison avec les planches 
seulement encrées : utilisée indifféremment pour les personnages et les décors, pour les ombres ou les matières, la teinte ne possède pas un rôle défini dans le système narratif. Certes, elle a souvent pour fonction de faire ressortir les personnages du fond, d'accentuer les volumes (on est en droit de penser à la fonction de l'éclairage au cinéma), mais la planche reste parfaitement lisible sans cet apport. De plus, Clowes inscrit généralement au niveau de l'encrage des indications de volumes, des ombres ou des distinctions entre les plans par l'épaisseur du trait, autant de dispositifs clarifiant les relations spatiales. En d'autres termes, cette couleur est avant tout un surcodage, un élément de renforcement des autres dispositifs graphiques. Elle participe de l'identité visuelle de GWGN, puisque la technique est plus rare que le simple noir et blanc, mais peut être altérée, voire supprimée, sans affecter profondément l'œuvre.

La situation est toute autre dans le film, qui investit pleinement ce domaine formel. Sin City a prouvé que la formule noir et blanc plus couleur d'appoint était possible au cinéma, mais en démontrant aussi à quel point un tel procédé devenait ostentatoire. Si le noir et blanc, éventuellement rehaussé, est un des modes établis de la bande dessinée d'auteur (voir le travail du créateur canadien Seth, par exemple), un attribut non remarquable, la couleur est la seule option transparente dans le cinéma dominant contemporain. Le contraste entre les deux versions dans ce domaine découle donc d'abord d'un écart de norme entre leur champ de production respectif. Cependant, le traitement de la couleur dans le film ne relève pas d'un simple naturalisme assimilable au « degré zéro de l'écriture » de Barthes.

Il est d'abord tentant d'y chercher un code. Le début du film suggère en effet une opposition possible entre le rouge des robes des diplômés et les teintes fluorescentes de jeunes danseuses de hip-hop. Le rouge est assimilé à plusieurs reprises au danger ou à l'interdit, de façon assez classique, mais il est difficile de parler de codage, faute de systématisation du procédé. De la même manière, si Rebecca est régulièrement vêtue de vert, l'effet n'est pas assez soutenu pour faire sens. Citons encore l'exemple de Roberta, professeur d'arts plastiques jouée par Illeana Douglas, aux cheveux roux, vêtue d'une tunique violette et plus tard d'un justaucorps rouge-vif, filmée sur fond orange sans explication à cette débauche chromatique. L'omniprésence de ces couleurs nonnaturalistes suggère que celles-ci doivent être organisées selon un code signifiant, comme le veut d'ailleurs la tradition hollywoodienne (Bourget 116-117), mais ce sens $r$ reste initialement obscur. Tout au plus cet usage d'une palette très vive permet-il, comme dans la bande dessinée, de distinguer nettement le premier et l'arrière-plan, soit par le contraste entre les couleurs choisies, soit par la différence de saturation et de luminosité.

Le sens de cette palette n'apparait qu'après trente-et-une minutes du film, soit la fin de son premier acte. Enid et Rebecca ont initialement donné un pseudo-rendez-vous romantique à Seymour et ont observé sa déconfiture. Voulant en savoir plus, elles se sont ensuite rendues chez lui et Enid lui a acheté un vieux disque de blues, sans intention de l'écouter. Peu après, elle tente d'expliquer à Rebecca ce que lui a inspiré cet achat, mais n'y parvient pas.

E: "In a way, he's such a clueless dork that he's almost cool"

R: “That guy is many things, but he definitely isn't "cool” [...]

E: "Yeah, but... you know what I mean"

R: "Not really..."

E: "Forget it, I can't explain it..." 

comme pour un double-take décalé. La caméra commence alors à tourner lentement autour de son visage, pour un plan qui rompt avec la frontalité implacable qui précède. Elle révèle alors la jeunesse de l'interprète, jusqu'alors dissimulée par ses tenues, ses coiffures et son sens de la répartie. Dans la lumière adoucie, la scène comprend un très gros plan sur le tourne-disque brun, noir et imparfait, soulignant sa matière et sa matérialité contre tous les signes d'artificialité et de superficialité accumulés jusqu'alors. Un fondu avec un effet d'analogie et de continuité visuelle entre le disque et le corps d'Enid souligne encore le lien établi entre la jeune fille et la musique. Cependant, le changement de palette est particulièrement perceptible au sein de ce dispositif, puisqu'il n'est pas seulement pris dans le système linéaire du récit. Il ne constitue pas une nouveauté, comme peut l'être le morceau musical, mais un changement de vision sur du déjà connu. Une palette assez proche de celle-ci, faite de brun et de gris, mais dans une ambiance diurne, était déjà utilisée au moment de l'achat du disque. Cependant, cette palette était moins remarquable puisqu'il n'y avait pas de recoloration de l'espace, et l'effet rhétorique évident était celui d'opposition: Steve Buscemi, homme médiocre aux couleurs aussi ternes que celles des figurants dans la salle d'arts plastiques (sa voiture est aussi brune et grise), était ainsi distingué des deux jeunes filles.

32 Il y a donc dans cette scène de l'écoute un point de basculement, qui assigne une valeur narrative non à une couleur unique, mais à une gamme de teintes. Le caractère acidulé, artificiel et solaire des couleurs de la première partie se comprend par contraste comme une manifestation de l'enfance et d'une modernité agressive. Sous sa forme la plus simple, le jeu sur la couleur vient donc renforcer la gamme d'oppositions entre le pôle Rebecca et le pôle Seymour. La tentative de rencontre entre les deux est de toute évidence un échec (Rebecca résume cette rencontre en affirmant à Enid: "I totally, totally, totally hate you » 36 minutes), et c'est donc dans la représentation d'Enid que les changements chromatiques se manifestent. Ses tenues s'assombrissent, perdent de 
leur contraste (et de leur couleur), pour ne retrouver leur exubérance que brièvement : sous la forme d'un uniforme de travail, pour une expérience de vendeuse de pop-corn, en salle d'arts plastiques ou dans un ultime rapprochement avec Rebecca. Ce n'est que lorsque Enid se brouille avec Seymour qu'elle est montrée portant un haut noir et jaune-vif ; en sa présence, ses vêtements sont le reste du temps sombres et discrets.

Les couleurs et la lumière offrent à Zwigoff et à son directeur de la photographie l'occasion d'introduire brièvement dans le cours du film des références à d'autres œuvres et d'autres codes : en ménageant une allusion visuelle à l'affiche de Pulp Fiction de Quentin Tarantino (1994), par exemple, ou en utilisant un éclairage froid et des couleurs bleutées pour introduire dans le film un univers qui lui est étranger. Dans cette scène, Seymour observe son amie dans son univers professionnel: avant même qu'il ne rompe avec elle, les couleurs démontrent leur incompatibilité. Le dialogue de la scène a d'ailleurs été considérablement raccourci par rapport au script (scène 92), couleur et lumière contextualisant la rupture sans rendre nécessaire une verbalisation complète.

C'est aussi la couleur qui contrôle notre lecture de la conclusion du film. Enid a alors opté pour une tenue rouge profond. Cette couleur est également celle des draps de Seymour, couvrant le lit dans lequel ils sont montrés après l'amour (84 minutes 40 ), exemple de codage chromatique classique ; il n'est peut-être pas anodin de noter que l'acte lui-même est élidé par un fondu au noir, le même procédé qui avait servi à coder l'écoute du disque plus tôt dans le film. Ce rouge profond n'est plus celui des robes universitaires de la première scène: il représente un compromis entre les couleurs primaires arborées par Rebecca et Enid dans la première partie du film, entre les murs roses récurrents de la ville et entre l'univers d'ombres et de couleurs froides de Seymour. L'indécision d'Enid se lit dans ce choix. Cependant, plus que son choix vestimentaire, c'est la transformation d'un espace déjà connu qui, comme lors de la scène de l'écoute, retient l'attention. L'arrêt de bus est un lieu récurrent dans le film, mais toujours montré de jour, à côté d'un mur d'un rose éblouissant: dans cette dernière scène, la nuit tombée transforme ce rose en mauve délicat, puis en rouge profond. L'éclairage indirect du lampadaire et d'une vitrine projette des ombres longues, et révèle des aspérités inattendues, mais c'est un univers de nuit bleutée qui domine le plan. C'est plus vrai encore dans l'ultime plan du film, où seuls deux phares rouges et quelques taches de lumière jaune rappellent la robe d'Enid et l'univers solaire du début du film.

La scène est très similaire à celle présentée dans GWGN (80), bien que le film l'inclue dans un montage alterné avec une scène montrant Seymour au restaurant. Le dernier plan, en particulier, est proche dans son cadrage et sa perspective de celui choisi par Clowes. Pourtant, le jeu des couleurs lui donne un sens différent, tant la palette nocturne a été associée à Seymour au long du film. Sa relation avec Enid est un échec, mais cette dernière part dans un univers transfiguré par cette rencontre. Les couleurs séduisantes de ce dernier plan ne sont pas exactement celles associées à Seymour, car si les nuances et les ombres lui appartiennent, le rouge, le jaune et le bleu sont les couleurs d'Enid. Ces teintes hopperiennes renvoient donc aussi à la personnalité de créatrice et d'illustratrice d'Enid, bien plus développée dans le film que dans la bande dessinée. Ni Rebecca, ni Seymour, qui collectionne mais ne crée pas, ni même Roberta (la professeur d'arts plastiques) pour qui le discours prime sur la création, ne sont 
présentés comme disposant de cette capacité à transformer le réel en expérience esthétique.

L'introduction de la couleur et de la musique signalent un élargissement du spectre de cette expérience esthétique. Film bavard, GWF est pourtant moins défini par le verbe que ne l'est la bande dessinée. Au contraire, les maîtres de la parole (psychologue, plasticienne) y sont constamment moqués. Dans la bande dessinée, l'angoisse naît de ne pas être sûr de ce que quelqu'un a dit (46), la rupture entre Enid et Rebecca se cristallise autour d'un mauvais choix de mot (58), et la raréfaction de la parole sert à conférer aux quelques mots prononcés dans l'épilogue une puissance indéniable (79-80). La scène finale de GWF oppose au contraire le silence et l'esthétique au verbe débordant et humiliant de la psychologue.

\section{Conclusion}

Les deux versions de Ghost World ont beaucoup en commun. Les deux cuvres se rejoignent ainsi dans leur humour cruel et leur mélancolie, dans la trame générale du récit, par la présence commune de certaines scènes, de pans entiers de dialogue, par la description de l'éloignement progressif de deux amies. Sur le plan des options de représentation et du style, des correspondances existent, bien que les deux objets culturels ne soient pas positionnés de façon identique au sein de leur champ respectif. Les caractéristiques de l'univers mis en place par Clowes informent en partie la mise en scène du film. Pourtant, ces points de grande proximité ne font que souligner l'écart entre les deux versions dans d'autres domaines. Zwigoff explore en réalité tout ce qui constitue la marge du récit d'origine. Il investit ainsi le champ de la couleur, cette dimension "joyeuse, légère comestible du cinéma " (Leutrat 129). Il développe le personnage de Seymour, qui n'existe même pas en tant que tel dans le roman graphique. Il inclut enfin dans le récit des épisodes annexes dus à Clowes mais n'appartenant pas à Ghost World, en adaptant aussi bien le péritexte que le récit luimême. Il s'agit moins d'un étoffement, que d'un redéploiement du récit, puisque ces éléments déplacent ou annulent certaines des traits du roman graphique. A la sécheresse et à l'ascétisme du travail de Clowes, à la construction de son «monde fantôme " se substitue ainsi une expérience visuelle plus séduisante, plus diversifiée, inscrite dans un discours sur l'esthétique relayé par divers personnages. L'objective laideur du monde de Clowes laisse place à un monde finalement attirant, malgré ses aspects dérisoires, à l'image des deux actrices principales du film. La fuite finale d'Enid ne se lit ainsi plus seulement comme l'aboutissement d'une résignation, mais aussi comme un acte créateur.

\section{BIBLIOGRAPHIE}

Bordwell, David, Janet Staiger et Kristin Thompson. Classical Hollywood Cinema, Film Style \& Mode of Production to 1960. New York : Routledge, 1988. 
Bourget, Jean-Loup. « Vertige du technicolor. » in La couleur en cinéma. Ed. Jacques Aumont. Paris : Cinémathèque française, 1995. 110-119.

Clowes, Dan. Caricature. Seattle, WA : Fantagraphics Books, 1998.

---. Ghost World, Special Edition. 1998. Seattle, WA: Fantagraphics Books, 2008.

---. Like a Velvet Glove Cast in Iron. Seattle, WA: Fantagraphics, 1993.

---. Lloyd Llewellyn. Seattle, WA: Fantagraphics, 1994.

---. Twentieth Century Eightball. Seattle, WA: Fantagraphics, 2001.

---. Wilson. Montréal: Drawn \& Quarterly, 2010.

Fresnault-Deruelle, Pierre. « Du Linéaire au Tabulaire. » Communications 24 (1976) : 7-23.

Gabilliet, Jean-Paul. « Du comic-book au graphic novel : l'européanisation de la bande dessinée américaine. » IMAGE [\&] NARRATIVE: ONLINE MAGAZINE OF THE VISUAL NARRATIVE, $\mathrm{N}^{\circ} 12$ (August 2005) 24 février 2011

<http://www.imageandnarrative.be/inarchive/tulseluper/gabilliet.htm>.

Groensteen, Thierry. Système de la bande dessinée. Paris: PUF, 1999.

Hatfield, Charles. Alternative Comics: an Emerging Literature. Jackson: University of Mississipi Press, 2005.

Hignite, Todd. In the Studio. Visits With Contemporary Cartoonists. New Haven and London: Yale University Press, 2006.

Hutcheon, Linda. A Theory of Adaptation. New York and London: Routledge, 2006.

King, Geoff. American Independent Cinema. New York and London: I.B. Tauris, 2009.

Kurtzman, Harvey (sc.) et Jack Davis, « Supermarkets » Mad 19 (janvier 1955): n.n.

Labarre, Nicolas. « Two Flashes. Entertainment, Adaptation: Flash Gordon as Comic Strip and serial. » Comicalités. 1 July 2011. 26 October 2011

$<$ http://comicalites.revues.org/249>.

Lefèvre, Pascal. « Incompatible Visual Ontologies. » In Film and Comic Books. Eds Gordon, Jancovish and MacAllister. Jackson: University Press of Mississipi, 2007. 1-12.

---. « Narration in Comics. » IMAGE [\&] NARRATIVE: Online Magazine of the Visual Narrative, $\mathrm{n}^{\circ} 1$.

August 2000. 26 october 2011

< http://www.imageandnarrative.be/inarchive/narratology/narratology.htm>.

Leutrat, Jean-Louis. « De la couleur-mouvement aux couleurs fantômes. » La couleur en cinéma. Ed. Jacques Aumont. Paris : Cinémathèque française, 1995. 126-36.

Peeters, Benoit (sc.) et François Schuiten (d.). Brüsel. Tournai : Casterman, 1992.

Schneider, Greice. « David Boring - Daniel Clowes. » The Comics Grid. 17 March 2011. 1 July 2011

<http://www.comicsgrid.com/2011/03/david-boring-daniel-clowes/>.

Staiger, Janet. « Independent of what? Sorting out differences from Hollywood. » In American Independent Cinema: Indie, Indiewood and Beyond. Eds. Geoff King, Claire Molloy, Yannis Tzioumakis. New York: Routledge, 2013.

«Subtlety gap can be hard to fill. » Birmingham Post (19 November 2001): 12.

Venezia, Tony. « Ghost World[s] and Non-Places. » The Comics Grid. 21 Mach 2011. 1 July 2011 <http://www.comicsgrid.com/2011/03/ghost-worlds-and-non-places-2/>. 
Ware Chris. Jimmy Corrigan, the Smartest Kid on Earth. New York: Pantheon Books, 2000.

American Beauty, dir. Sam Mendes, Dreamworks Pictures, 1999.

Dick Tracy, dir. Warren Beatty, Touchtstones Pictures, 1990.

Ghost World, dir. Terry Zwigoff, Icon Entertainment, 2001.

Halloween, dir. John Carpenter, Compass International Pictures, 1978.

Horse Whisperer (The), dir. Robert Redford, Touchstone Pictures, 1998.

Hulk, dir. Ang Lee, Universal Studios, 2003.

Lost in Translation, dir. Sophia Coppola, American Zoetrope et Tohokushinsha Film, 2003.

Pulp Fiction, dir. Quentin Tarantino, Miramax, 1994.

Rear Window, dir. Alfred Hitchcock, Universal Studios, 1954.

Scott Pilgrim vs. the World, dir. Edgar Wright, Big Talk Films, 2010.

Sin City, dir. Roberto Rodriguez et Frank Miller, Troublemaker Studios, 2005.

Timecode, dir. Mike Figgis, Screen Gems, 2000.

\section{NOTES}

1. Pour éviter la confusion entre ces deux objets culturels homonymes, il sera fait référence dans la suite de ce texte au roman graphique via l'abréviation GWGN. Les références parenthétiques renvoient à la pagination de la dernière édition en date, Ghost World, Special Edition, citée dans la bibliographie. Le film sera quant à lui noté GWF.

2. Ces courts récits ont depuis été rassemblés dans deux recueils, Twentieth Century Eightball et Caricature.

3. C'est-à-dire une bande dessinée produite en dehors des deux géants du secteur, DC et Marvel Comics, spécialisés dans le genre super-héroïque. Charles Hatfield propose de distinguer bande dessinée indépendante et bande dessinée alternative, mais note que les deux termes sont le plus souvent utilisés de façon interchangeable (Hatfield 26).

4. S'il n'existe pas une définition unique du cinéma indépendant, la question $d u$ modèle industriel reste centrale (King 2 ; Staiger 21-24). Or, les économies des deux secteurs sont très différentes, ne serait-ce que parce qu'en bande-dessinée, l'éditeur concentre le plus souvent les rôles dévolus au producteur et au distributeur dans le cinéma nord-américain. Dès lors, on ne peut parler que de ressemblances thématiques (méfiance envers le genre, insistance sur l'auteur) entre cinéma indépendant et bande dessinée indépendante.

5. Le script inclus dans cette édition, différent par endroits de la version filmée, constitue en ce sens une troisième variation, qui s'ajoute au roman graphique et au long-métrage. 


\section{RÉSUMÉS}

Ghost World, le roman graphique de Dan Clowes (1998) se caractérise par la mise en place d'un espace géométrique et hostile, sans pour autant renoncer à une certaine transparence du médium de la bande dessinée. Ce texte examine dans un premier temps la constitution de cet espace, par un équilibre entre un usage classique et un usage plus rhétorique ou expressif de certains dispositifs propres à la bande dessinée (format des cases, rôle de la bulle, rôle de la grille). Dans un second temps, il s'agit de repérer comment le cinéaste Terry Zwigoff mobilise des dispositifs proprement cinématographiques pour transcrire une partie de ces effets dans sa propre version de Ghost World (2001). L'usage de la couleur, en particulier, apparaît comme un facteur distinguant les deux versions et produisant des effets de sens très différents.

Ghost World, Dan Clowes's 1998 graphic novel, features a geometrical and hostile diegetic space, yet does not forego a classical system of comics, which could be characterized by the notion of "transparency". This article focuses first on the way this balance is achieved, through the use of formal devices specific to the medium, from the grid to the size of the panels to the role of speech balloons. A second part examines the solutions Terry Zwigoff used to recreate the effects of these formal devices through specifically cinematographic techniques, when he adapted Ghost World into a film in 2001. The use of color, in particular, distinguishes the graphic novel from its adaptation, leading to different readings of a similar story.

\section{INDEX}

Mots-clés : linéaire, tabulaire, adaptation, grille, couleur, géométrie

Keywords : linear, tabular, adaptation, grid, color, geometry

\section{AUTEURS}

\section{NICOLAS LABARRE}

Université Michel de Montaigne, Bordeaux 3

Maître de conférences en civilisation américaine

Nicolas.labarre@u-bordeaux3.fr 